\title{
Different Types of Myotonic Muscle Disorders at Dhaka Medical Collage Hospital
}

\author{
S DAISYa ${ }^{\mathrm{a}}$ SMNM SAYEED ${ }^{\mathrm{b}}$, QD MOHAMMAD ${ }^{\mathrm{c}}, \mathrm{S} \mathrm{HOSSAIN}^{\mathrm{d}}, \mathrm{MHABIB}^{\mathrm{e}}$, \\ A HOQUE $^{\text {f }}$ B ALAMg, B HAQUE ${ }^{\text {h }}$
}

\author{
Summary: \\ After introduction of EMG at Dhaka Medical Collage \\ on January 01, 2006 , a total of 329 cases referred to \\ EMG laboratory for electrophysiologic evaluation over \\ a period of one \& half year (January 01, 2006 to June \\ 30, 2007). Among them, 45 cases diagnosed as different
}

\section{Introduction:}

Before EMG was used in Bangladesh, common myopathies were Duchenne muscular dystrophy (DMD) \& sometimes Myotonic dystrophy (MD). But in developed countries in addition to these two myopathies varieties of other myopathies are commonly diagnosed. So it was thought that myopathies were more common in developed countries than ours. But with the introduction of EMG \& NCS in Bangladesh, we now come across varieties of myopathies as in developed countries.

So electrophysiology plays a key role along with muscle biopsy for diagnosing myopathies ${ }^{1}$. EMG can often confirm that a myopathy is present and can add diagnostic information if certain types of spontaneous activities were present. For instance fibrillation and positive waves in myopathy suggests the possibility of inflammatory or necrotic features, whereas myotonic discharge suggests one of the myotonic muscle disorders such as acid maltase deficiency, myotubular myopathy, certain toxic myopathies or myotonic dystrophy.

a. Dr. Selina Daisy, MD. (USA), b. Dr. S.M. Nurul Momin Sayeed, M.B.B.S, c. Professor Dr. Quazi Deen Mohammad, FCPS (Medicine), MD (Neuro), Fellow Neurology (America), d. Dr. Sakhawat Hossain, FCPS (Medicine), MD (Neurology), e. Dr. Mansur Habib, FCPS (Medicine), MD (Neurology), MRCP (UK), FRCP (Edin), f. Dr. Azharul Hoque, FCPS (Medicine), MD (Neurology), g. Dr. Badrul Alam, MD (Neurology), h. Dr. Badrul Haque, M.B.B.S, Dip in Neuro, Dhaka Medical College, Dhaka.

Address of Correspondence: Dr. Selina Daisy, MD. (USA), Associate Professor of Neurology, Department of Neurology, Dhaka Medical College, Dhaka, Bangladesh. Mailing address: daisyselina@yahoo.com.

Received: 9 August, 2007

Accepted: 21 May, 2008 types of myopathies. Among these cases, eleven (11) diagnosed as myotonic muscle disorders. The subtypes of myotonic muscle disorders were 1. Myotonic Dystrophy. 2. Proximal Myotonic Myopathy. 3. Myotonia Congenita

(J Bangladesh Coll Phys Surg 2008; 26: 121-123)

The myotonic muscle disorders comprise a group of disorders characterized by muscle stiffness, pain and sometimes weakness, which may be intermittent or constant. Evaluation of these disorders in the electromyography (EMG) laboratory is particularly gratifying as the EMG accompaniment of myotonia is easily recognized. These myotonic discharges produce a distinctive " dive-bomber" sound on EMG.

\section{Materials and Methods:}

This study was conducted at EMG laboratory under Neurology Department of Dhaka Medical Collage. Study period was one year six month (January 01, 2006 to June 30, 2007). Both inpatient and outdoor patient were referred to EMG laboratory with various diagnoses.

All patients' clinical information along with some laboratory data were collected and stored in Excel program. This study was retrospective. The data was reviewed from the Excel program.

Routine nerve conduction study (NCS) and needle electromyogram (EMG) were done in all patient.

After history was taken and a directed physical examination performed, a nerve conduction study (NCS) was done followed by needle electromyogram (EMG).

Needle EMG cannot be planned or properly interpreted without knowledge of underlying nerve.

Peripheral nerves can usually be easily stimulated and brought to action potential with a brief electrical pulse applied to the overlying skin.

Nerve selected for study depends on the distribution of the patient's symptoms, sign and the differential diagnosis. 
Motor and sensory nerves were studied by stimulating the nerve with the recording electrodes placed over the distal muscle or the cutaneous sensory area.

After nerve conduction study (NCS), needle electromyography (EMG) was done.

Like the NCS, each needle EMG study was individualized, based on the clinical findings and the differential diagnosis.

After selecting a muscle to be studied, proper needle insertional point was identified, and the needle was then quickly inserted through the skin into the muscle.

The patient is then asked to activate the muscle and then asked to relax the muscle. Then the needle was withdrawn.

\section{Results:}

A total of 329 patients were referred to EMG laboratory with various types of clinical diagnosis ranging from diseases of nerve, neuromuscular junction (NMJ) disorder to muscle diseases.

Among these, 45 patients were diagnosed as myopathies of various types. Among these 11 patients were diagnosed as myotonic muscle disorders.

The subtypes of myotonic muscle disorders were-

Myotonic dystrophy : 6

Proximal myotonic dystrophy: 3

Myotonia congenita : 2

\section{Discussion:}

In the present study, six cases of myotonic dystrophy were found. Among these 6 cases, 4 were diagnosed clinically and confirmed by EMG. Two cases of myotonic dystrophy were missed clinically. Two cases of myotonia congenita were diagnosed clinically as also myotonic dystrophy. Myotonic dystrophy is among the most common of the myotonic muscle disorder. This is an inherited (autosomal dominant) multisystem disease. Age of onset is variable. Symptoms may appear at any time from birth to old age ${ }^{2}$. All patients presented in middle age, i.e. $>25$ years. Rate of deterioration was commonly slow, with little change over long period.
Some people never had significant muscular disability. Four out of six patients were in this group. Face, jaw \& neck area are involved. Large weight bearing muscle of legs and thighs are much less affected $^{3}$. Muscle stiffness or myotonia is characteristic, especially affecting the hands ${ }^{4}$. All four patients had difficulty in releasing their hand grip and also had percussion myotonia over the thenar eminence. This differentiates from proximal myotonic dystrophy \& myotonia congenita, where myotonias are more proximally involved 4.5. Involvement of other body system is frequent such as cataract, disturbances of heart rhythm, hormone problem, child bearing difficulties and dysphagia ${ }^{2}$. One of the clinically missed patients had dysphagia, which was so severe that she needed nasogastric tube for feeding and her fingers were so myotonic and weak that she had constantly adducted thumb with other fingers were extended and adducted. She could not use her fingers or grab any thing. She was diagnosed clinically as neuromuscular junction (NMJ) disorder or chronic inflammatory demyelinating polyneuropathy (CIDP). The other patient which was also missed clinically had same problem of her hand like the other patient but without dysphagia. cataract was found in 4 patients. Investigation for other problem was not done, the other multisystem involvement could not be identified. Gene for myotonic dystrophy is on chromosome 19 but it is variable in extent, even in a single family, because it is unstable. The length of a particular 'triplet repeat sequence' (CGT) is expanded in myotonic dystrophy and this may vary from single expansion in mildly affected individual to a very large one in severely affected patients ${ }^{3}$. Gene study was not done.

All cases of proximal myotonic myopathy (PROMM) were missed clinically. These were diagnosed as NMJ disorder, polymyositis, functional or limb girdle muscular dystrophy.

Proximal myotonic myopathy (PROMM) is also known as DM 2. PROMM has been defined recently 5 . It is rather similar to but distinct from myotonic dystrophy ${ }^{6}$. Here myotonia is less prominent rather muscle pain, stiffness \& fatigue is more common. Development of proximal lower extremity weakness is more common than hands \& lower legs as in 
myotonic dystrophy. Parts close to trunk of body i.e. neck, shoulder, hip flexor \& upper leg are mostly affected than face, jaw muscle or ptosis as in myotonic dystrophy. Smooth muscles are sometime affected i.e. dysphagia. All of 3 patients had most of the criteria with variable expression. Molecular genetic testing of the CTG trinucleotide repeat expansion is a reliable diagnostic method in myotonic dystrophy, which is normal in proximal myotonic myopathy. And no genetic test so far available ${ }^{5}$.

Myotonia congenita were both from same family. They came with diagnosis of myotonic dystrophy since they had mild myotonia in their hands. Clinically myotonia congenita have one characteristic finding of pronounced muscle development despite lack of exercise. No clinician will miss it if they have come across only with one patient of myotonia congenita.

Clinician can easily come to a definite diagnosis of various types of myotonic myopathy instead of diagnosis 'functional' or NMJ disorder and putting patients with unnecessary medicine and stress.

\section{Conclusion:}

Like many developed countries, various types of myopathies, especially various types of myotonia are found in Bangladesh \& can easily be diagnosed clinically \& confirmed by EMG.

\section{Acknowledgement:}

We are thankful to the principal of Dhaka Medical Collage \& the Director of Dhaka Medical Collage Hospital for allowing us to publish this study. We are also thankful to the concerned family patient for their cooperation.

\section{References:}

1. Preston DC, Shapiro BE, Electromyography and Neuromuscular disorders 2005 Elsevier ButterworthHeinemann, 591-600.

2. Sir Peter S. Harper, Myotonic Dystrophy: The facts: A book for patients \& Families (Oxford Medical Publication) Oxford University Press. ISBN O-19-852586-9.

3. www-dystrophy org/information-resources.

4. Daisy S, Mohammad QD, Haque A, Alam B, Myotonia congenita in a Bangladeshi family ( A case report). J Bang coll Phy \& Surg. 2005; 23 (3): 143-145.

5. Riker K, Myotonic dystrophy and proximal myotonic myopathy, J. Neurol. 1999; 246: 334-338.

6. Myotonic dystrophy: From Wikpedia, the free encyclopedia

7. Bradley W, Daroff R, Fenichel G, Jamkovic J, (editors). Neurology in clinical practice. Fourth edition. Butterworth Heinemann, 2004; 2483-2486. 